Empirical evidence shows that high concentrations of the disaccharide, trehalose, in the tissues of certain microorganisms, insects, and desert plants allows them to survive in a state of suspended animation under conditions of water deficiency. Trehalose seems to work by replacing the water shell around macromolecules such as proteins and lipid membranes. No vertebrate organism has yet been found that can either synthesize trehalose or survive desiccation. In this issue, Levine and colleagues infect cultured human

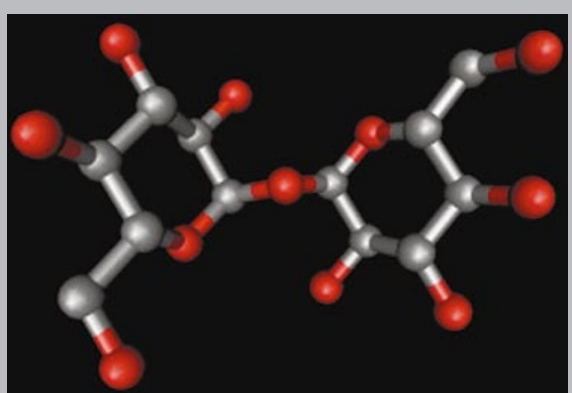
cells with an adenovirus expressing the genes encoding the trehalose biosynthetic enzymes from bacteria (see pp. 168 and 145). The infected cells produced trehalose, and tolerated the complete lack of free water for up to five days with only a moderate reduction in viability. These results may lead to new ways of storing or transferring human cells, tissues, and organs.

In a related manuscript, Toner and coworkers (p. 163) show that intracellular trehalose can also significantly enhance the survival of mammalian cells following cryopreservation. Instead of engineering the cells to synthesize trehalose, they exploited the ability of a recombinant protein, $\alpha$-hemolysin, to insert spontaneously into membranes and create small pores. In an earlier study, these workers engineered the protein such that its pore would open and close in response to the absence or presence of zinc ions in the medium, respectively. Cells treated with the modified protein were bathed in trehalose with the pores opened just long enough to load the cells with trehalose, before they were plunged into liquid nitrogen. Cells treated this way were much more likely to survive the deep-freeze than cells that were either untreated or exposed to the unmodified hemolysin and trehalose.

\section{Engineering better beans}

Soybeans are lauded for the health benefits they provide to Asian diets. These can be traced, at least in part, to their high isoflavones content. These secondary metabolites are found almost exclusively in legumes, and their levels in soybeans can vary quite a bit depending on growth conditions. Technologies for manipulating isoflavone levels are of keen interest as a means for producing beans with higher or more uniform isoflavone content, or for engineering non-leguminous crops to provide the health benefits of soybeans without the beans. McGonigle and colleagues now have taken an important step toward this goal. First they isolated the previously unidentified isoflavone synthase gene from soybeans and a number of other plants by functional expression of expressed sequence tags in yeast. They then introduced the soybean isoflavone synthase gene into the non-leguminous plant Arabidopsis, and showed that the plants then produced the isoflavone genistein (see p. 208).

\section{Technical reports}

Kolusheva et al. describe a colorimetric assay for identifying peptides with antimicrobial activity (see p. 225). They found that assemblies of phospholipids and polymerized polydiacetylene would change colors quite strikingly upon interacting with certain peptides. The ability of these peptides to produce the structural perturbations that led to the color change correlated with their antimicrobial activity, providing a more rapid way of screening peptides for this activity.

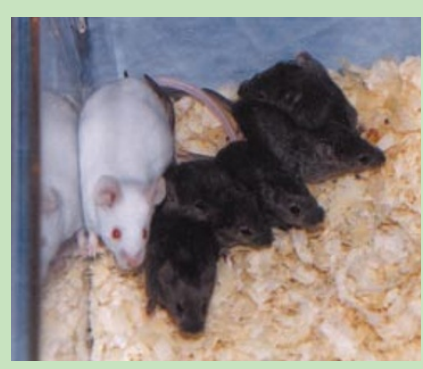

Oocyte enucleation is a critical parameter affecting the efficiency of cloning by nuclear transfer. It is currently performed by aspirating the cytoplasm just below the first polar body, followed by Hoechst staining to confirm the removal of DNA. In this issue, Keefe and colleagues describe a noninvasive technique for spindle imaging and oocyte enucleation using a new microscope, termed the Pol-Scope (p. 223). They show that they can achieve an enucleation efficiency of $100 \%$, and that the reconstituted rodent oocytes develop normally.

\section{To bind or not to bind}

Complementation of deletion mutants of $\beta$ galactosidase is a useful technique for detecting the interaction of cytoplasmic proteins. The interaction of protein domains, individually fused to two different, weakly complementing $\beta$-gal mutants, drives the association of the latter and reconstitutes enzyme activity. In this issue, Blau and coworkers have adapted this technology to monitor the ligand-dependent association of receptors within the two-dimensional space of the plasma membrane in living cells. They demonstrate that $\beta$-gal complementation provides a rapid, simple, and sensitive assay to monitor the kinetics of receptor dimerization, which may prove useful for screening chemical libraries for compounds that affect receptor action.

\section{A peptide mimetic of Herceptin}

Monoclonal antibodies that bind to the ectodomain of the $\mathrm{p} 185^{\mathrm{HER} 2 / \mathrm{neu}}$ oncoprotein have been shown to reduce the growth of certain kinds of tumors. Herceptin, a recombinant humanized form, now has been approved for use in humans. However, full-length antibodies can be costly to produce, limited in their ability to penetrate certain tissues, and can sometimes cause adverse immunological reactions in patients. As a way around these potential therapeutic problems, Murali and colleagues have used rational design to develop a peptide mimetic of Herceptin (see p. 194). The peptide mimic had activity that was comparable to that of the Herceptin antibody in reducing proliferation of cell lines overexpressing $\mathrm{p} 185^{\mathrm{HER} 2 / \mathrm{neu}}$, and in inhibiting in vivo tumor growth in nude mice.

\section{Next month in Nature Biotechnology:}

\section{Assaying kinase activity in single mammalian cells
Defining metabolic pathways within complex metabolic networks

Visualizing gene expression in vivo
Managing Bt-engineered crops

